\title{
Article \\ Monolithic Double Resonator for Quartz Enhanced Photoacoustic Spectroscopy
}

\author{
Roman Rousseau, Diba Ayache, Nicolas Maurin, Wioletta Trzpil (D, Michael Bahriz and Aurore Vicet *
}

Citation: Rousseau, R.; Ayache, D.; Maurin, N.; Trzpil, W.; Bahriz, M.; Vicet, A. Monolithic Double Resonator for Quartz Enhanced Photoacoustic Spectroscopy. Appl. Sci. 2021, 11, 2094. https://doi.org/ 10.3390/app11052094

Academic Editor: Bernhard Wilhelm Roth

Received: 27 January 2021

Accepted: 23 February 2021

Published: 26 February 2021

Publisher's Note: MDPI stays neutral with regard to jurisdictional claims in published maps and institutional affiliations.

Copyright: (c) 2021 by the authors. Licensee MDPI, Basel, Switzerland. This article is an open access article distributed under the terms and conditions of the Creative Commons Attribution (CC BY) license (https:// creativecommons.org/licenses/by/ $4.0 /)$.
IES, University Montpellier, CNRS, 34090 Montpellier, France; roman.rousseau@ies.univ-montp2.fr (R.R.); diba.ayache@ies.univ-montp2.fr (D.A.); nicolas.maurin@ies.univ-montp2.fr (N.M.); Wioletta.Trzpil@ies.univ-montp2.fr (W.T.); michael.bahriz@umontpellier.fr (M.B.)

* Correspondence: aurore.vicet@umontpellier.fr

Abstract: A new approach for Quartz Enhanced Photoacoustic Spectroscopy is presented, based on an acoustic excitation from the outside of the prongs of a quartz tuning fork, to increase the sensitivity of the sensor. For this purpose, we introduce a monolithic acoustic double-resonator (double-mR) in a T-shape configuration, using 3D printing. It was modelized and experimentally characterized using a $1392 \mathrm{~nm}$ distributed feedback laser diode, targeting a water vapor absorption line. The setup showed a two-factor enhancement of the signal, compared to a classical off-beam QEPAS approach and confirmed the strong interest of photolithographic printing techniques for acoustic developments.

Keywords: photoacoustics; infrared; laser; sensing; QEPAS

\section{Introduction}

Gas sensing is strongly demanding on sensitive, selective, and efficient techniques, to be implemented on laboratories experiments, environmental, medical, or industrial pur-poses. Since its invention in 2002 [1], Quartz Enhanced Photoacoustic Spectroscopy (QEPAS) demonstrated many advantages among techniques based on tunable sources and indirect gas absorption detection.

Photoacoustic spectroscopy relies on light absorption, through a specific gaseous species, when an absorption line corresponds to the emitted wavelength of a source. The source can be a laser or a broader source such as a light emitting diode [2]. While the source intensity is modulated, the light absorption is modulated as well, and gives rise to a localized sound wave at the modulation frequency. This indirect photonic effect is called the photoacoustic effect, which has been exploited for years [3], using resonant acoustic cells and microphones for sound sensing. In QEPAS, the sound wave interacts with a mechanical transducer (a quartz tuning fork or QTF), leading to a piezoelectric current. Thanks to the very high quality factor of the QTF ( 10,000 at atmospheric pressure), it is unnecessary to use any resonant acoustic cell, as done in classical microphone photoacoustics sensing.

In order to enhance the pressure wave amplitude, some small tubes called microresonators $(\mathrm{mR})$ can be added to the optical setup, leading to two main configurations (on-beam/off-beam). In the on-beam configuration [4], the resonator tubes are placed on both sides of the QTF plane, leaving a small air gap $(<100 \mu \mathrm{m})$ with the QTF prongs. In the off-beam configuration [5], the resonator tube is located on one side of the QTF, in the center alongside the prongs. On-beam QEPAS exhibits a good signal enhancement factor of around 30 [6,7], but it requires a stringent optical alignment to avoid photothermal ef-fects, due to direct illumination of the QTF. Standard commercial QTF are not adapted to long wavelength sources such as quantum cascade lasers because of the small gap $(0.3 \mathrm{~mm})$ between the prongs, leading to the development of large custom QTF, first for $\mathrm{THz}$ sensing [8,9], and their successful use in several applications. Despite a smaller signal enhancement, the off-beam configuration reduces optical constraints, gives more flexibility 
to the setup developments, and can be competitive in terms of sensitivity with on-beam QEPAS. This configuration was studied theoretically [10] and successfully implemented in molecular spectroscopy, for many purposes [11-15].

The two QTF prongs move symmetrically and are best coupled with a cylindrical acoustic wave, when the longitudinal axis of the wave is located in between the two prongs. Both on-beam and off-beam satisfy this condition-one acoustic source symmetrically acting on the inner surface of both prongs. Alternatively, the QTF can be excited by applying a force on the external surface of both prongs, but it requires two acoustic sources. We rethought the design of the spectrophone to be able to excite the QTF from the outside of the two prongs.

A comparable approach was realized at the same time by $\mathrm{Hu}$ and $\mathrm{Al}[16,17]$. This design was based on a QTF-embedded, double-pass, off-beam spectrophone. Similar to the original off-beam $\mathrm{mR}$ [5], the spectrophone is made of two cylindrical $\mathrm{mRs}$ with a slit in the center. The QTF is embedded in the mRs, the slit is large enough to partly immerse the QTF prong inside the $\mathrm{mR}$ cavity. In this configuration, two acoustic sources (from the two $\mathrm{mRs}$ ) were employed to excite the external surfaces of the prongs.

The novelty of our approach relied on a monolithic double-resonator (double $\mathrm{mR}$ ) in a T-shape configuration, 3D-printed (stereolithography = SLA) characterized, and modelized, thanks to our experience on single resonator evaluation [15]. In this paper, we pre-sent this new configuration, its design, characterization, and evaluation, using an open-air QEPAS configuration dedicated to ambient water vapor sensing with a $1392 \mathrm{~nm}$ DFB laser.

\section{Experimental Setup}

The QEPAS spectrophone was designed to excite the QTF from the outside of the prongs. We used two off-beam mRs placed in a mirror symmetry and the QTF located in between (Figure 1). The optical path was more complex, compared to a classical off-beam QEPAS approach. The laser beam went through one microresonator $(\mathrm{mR} 1)$, underwent two right angle reflections that brought it parallel to the incoming beam with a lateral shift $(\delta)$, and finally crossed the other microresonator ( $\mathrm{mR} 2)$.

In this configuration, one could consider two acoustic sources photogenerated with the same laser. In order to maximize the excitation of the QTF, the two acoustic sources must be in-phase. Considering two identical mRs and two identical mR-QTF gaps, the phase shift $\Delta \varphi$ between the two acoustic sources could be calculated with the optical path difference $\Delta L$, between the two mRs:

$$
\Delta \varphi=k \Delta L=1.3 \times 10^{-5} \mathrm{rad}
$$

With $k$ as the wave vector, $\Delta L=2.2 \mathrm{~cm}\left(2 \mathrm{~L}_{\mathrm{mR}}+\delta\right.$ the optical path between the $\left.2 \mathrm{mRs}\right)$. The phase shift was negligible; therefore, the $2 \mathrm{mRs}$ were considered to be acoustically in phase.

The dimensions of the monolithic double $\mathrm{mR}$ were chosen, after the characterization of several individual $\mathrm{mR}$. A set of many different $\mathrm{mR}$ with various dimensions, fabricated from aluminum micromachined blocks, were characterized following the setup described in [15]. We chose a large main hole $\left(R_{m R}=0.5 \mathrm{~mm}\right)$ to help the alignment process. The acoustic response of these individual mRs was studied using a photoacoustic signal generated by the absorption of a water line of a $1392 \mathrm{~nm}$ single frequency laser (https: / / eblanaphotonics. $\mathrm{com} /$ (accessed on 26 February 2021)). The laser was fully characterized, as shown in Figure 2 . 

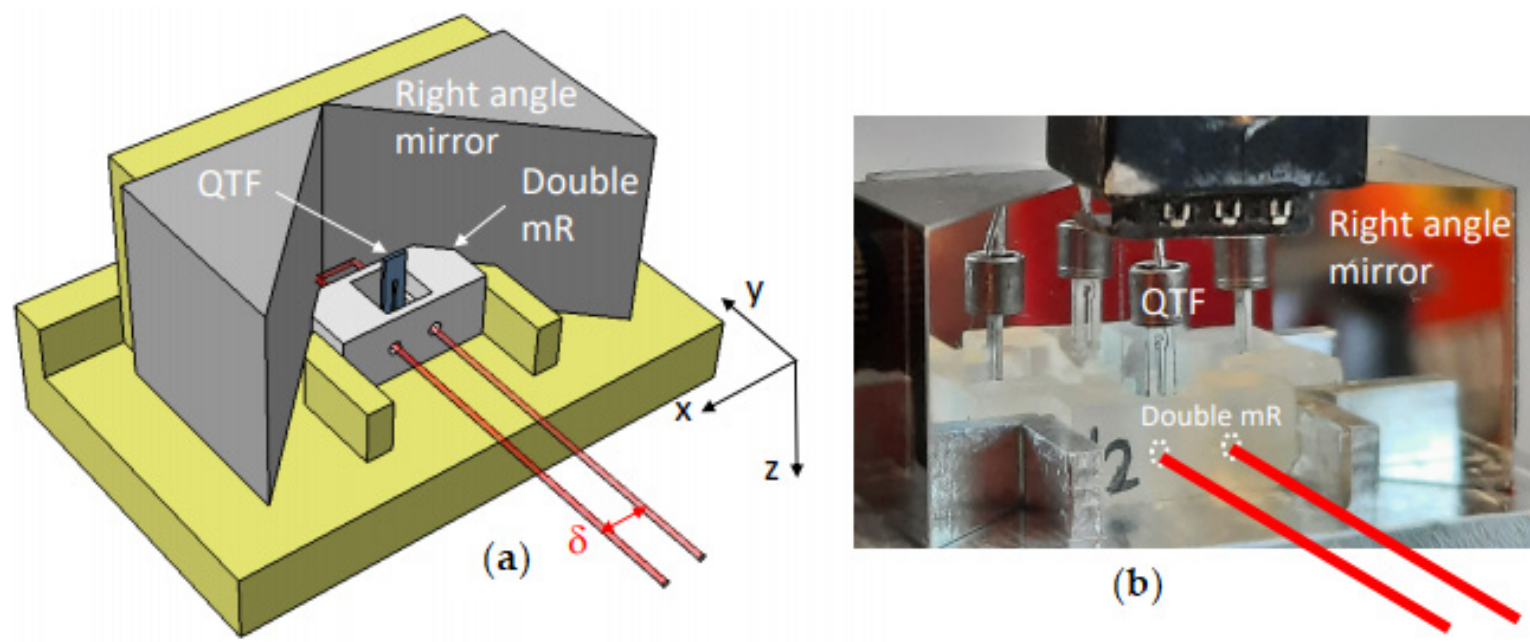

(c)

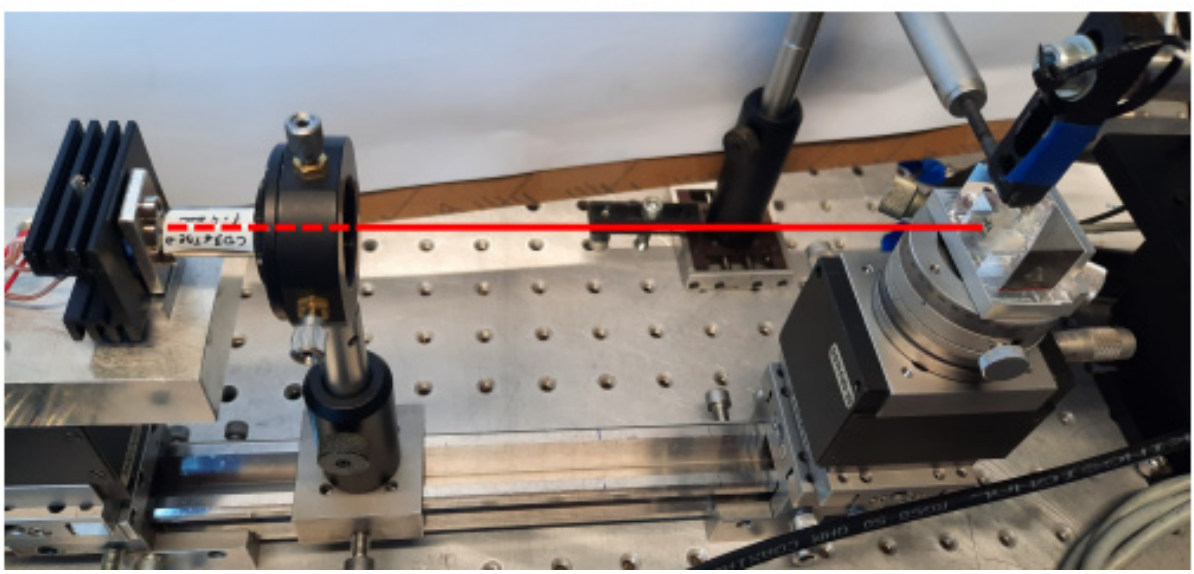

Figure 1. (a) Optical QEPAS setup of the double microresonator (double $\mathrm{mR}$ ) with the 2 right angle mirrors and the QTF position. (b) Picture of the setup. (c) Picture of the complete bench, including the laser, the focusing lens, and QEPAS spectrophone.

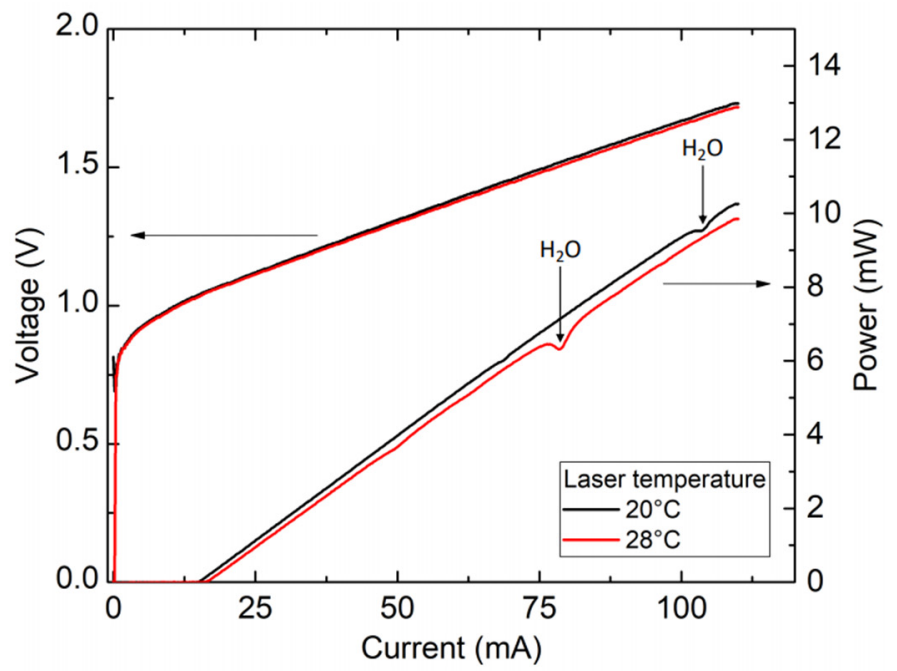

(a)

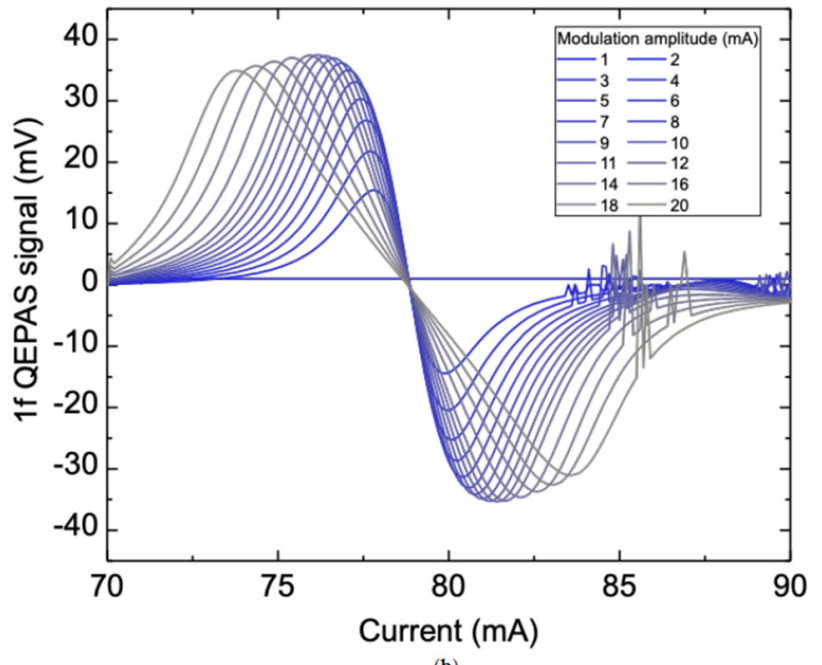

(b)

Figure 2. Characteristics of the EBLANA $1392 \mathrm{~nm}$ DFB laser. (a) LIV curves and (b) 1f-QEPAS response in an off-beam configuration with a single microresonator. The spikes at higher currents were due to insignificant experimental artefacts. The QEPAS response was obtained for a laser temperature of $28^{\circ} \mathrm{C}$ and ambient humidity of $50 \% \mathrm{RH}$. 
The target $\mathrm{H}_{2} \mathrm{O}$ line had a line strength of $1.5 \times 10^{-20} \mathrm{~cm}^{-1} /\left(\right.$ molecule $\left.\times \mathrm{cm}^{-2}\right)$, centered in $1392.54 \mathrm{~nm}\left(7181.18 \mathrm{~cm}^{-1}\right)$. The amplitude of acoustic wave, based on a given ambient water absorption (absolute humidity $\sim 1 \%$ in volume ratio), corresponded to an optical absorption coefficient of $1.15 \times 10^{-2} \mathrm{~cm}^{-1}$, which was relatively high, as compared to standard gas sensor experiments, but convenient for making a proof of concept.

We used a standard MEMS microphone (Knowles SPU0410LR5H-QB) to record the frequency response of the resonators [15]. These characterizations were conducted using mRs with lengths around $\lambda / 2$ for a $215 \mathrm{~Hz}$ acoustic wave, adjusted to the theoretical QTF resonant frequency. The best frequency-adjusted response was obtained for a length between 5 and $5.5 \mathrm{~mm}$ (Figure 3). We also fabricated 3D stereolithography printed $\mathrm{mRs}$ and noticed that changing the material did not modify the resonant properties-the quality factor $(Q=9$ for ref a9) and the resonant frequency remained the same. Following these results, we decided to choose $\mathrm{L}_{\mathrm{mR}}=5.3 \mathrm{~mm}$ to fit as properly as possible to the QTF resonant frequency.

\begin{tabular}{l|ccc} 
& $\boldsymbol{a 7}$ & $\boldsymbol{a 8}$ & $\boldsymbol{a 9}$ \\
\hline $\mathrm{R}_{\mathrm{mR}}(\mathrm{mm})$ & 0.5 & 0.5 & 0.5 \\
$\mathrm{~L}_{\mathrm{mR}}(\mathrm{mm})$ & 5 & 5.5 & 6
\end{tabular}
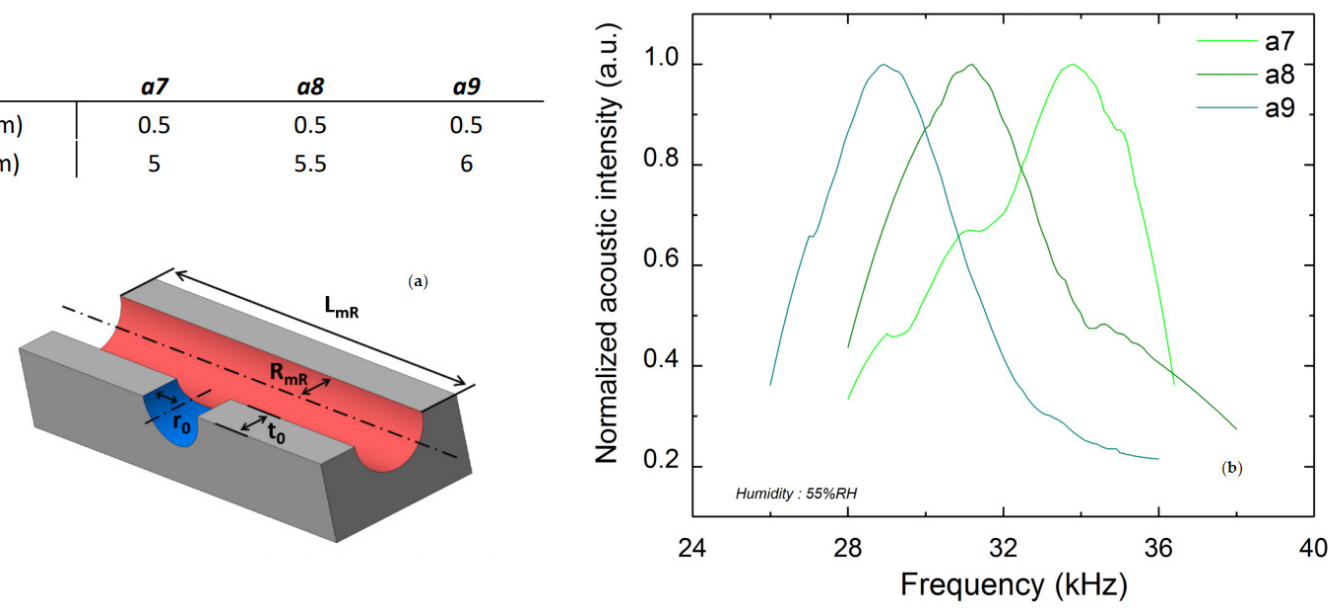

Figure 3. (a) Sectional view and dimensions of $3 \mathrm{mRs} a 7$, a8, and a9, sharing the same radius and different length. $\mathrm{r}_{0}=0.25 \mathrm{~mm}, \mathrm{t}_{0}=0.3 \mathrm{~mm}$. (b) Normalized experimental acoustic resonance of the 3 resonators.

In this double $\mathrm{mR}$ configuration, the two individual $\mathrm{mRs}$ were uncoupled, the acoustic wave was mainly confined in the main tube. Its amplitude quickly decreased out of the T tube, as can be seen in Figure 4. To evaluate the normalized sound distribution inside and outside the mRs, a thermo-acoustic frequency analysis of the pressure wave was realized by the finite element method (COMSOL). As predicted by analytical approaches [10,15], the wave was not entirely confined into the tube-about $30 \%$ of the total pressure extended outside due to the open ends effect and because of the T-shape.

The two mRs were accurately pre-positioned thanks to the monolithic design. They were spaced with a distance equal to the width of the QTF, plus an ideal gap of $70 \mu \mathrm{m}$ on each side [15]. The two mirrors were glued onto a plate, thus setting an angle of $90^{\circ}$ between the two reflective surfaces. The back plate slid along a shoulder in the back of the holder. Therefore, the two mirrors could be translated in the $x$ direction, offering a simple mean of adjusting the distance between the in/out beams. 

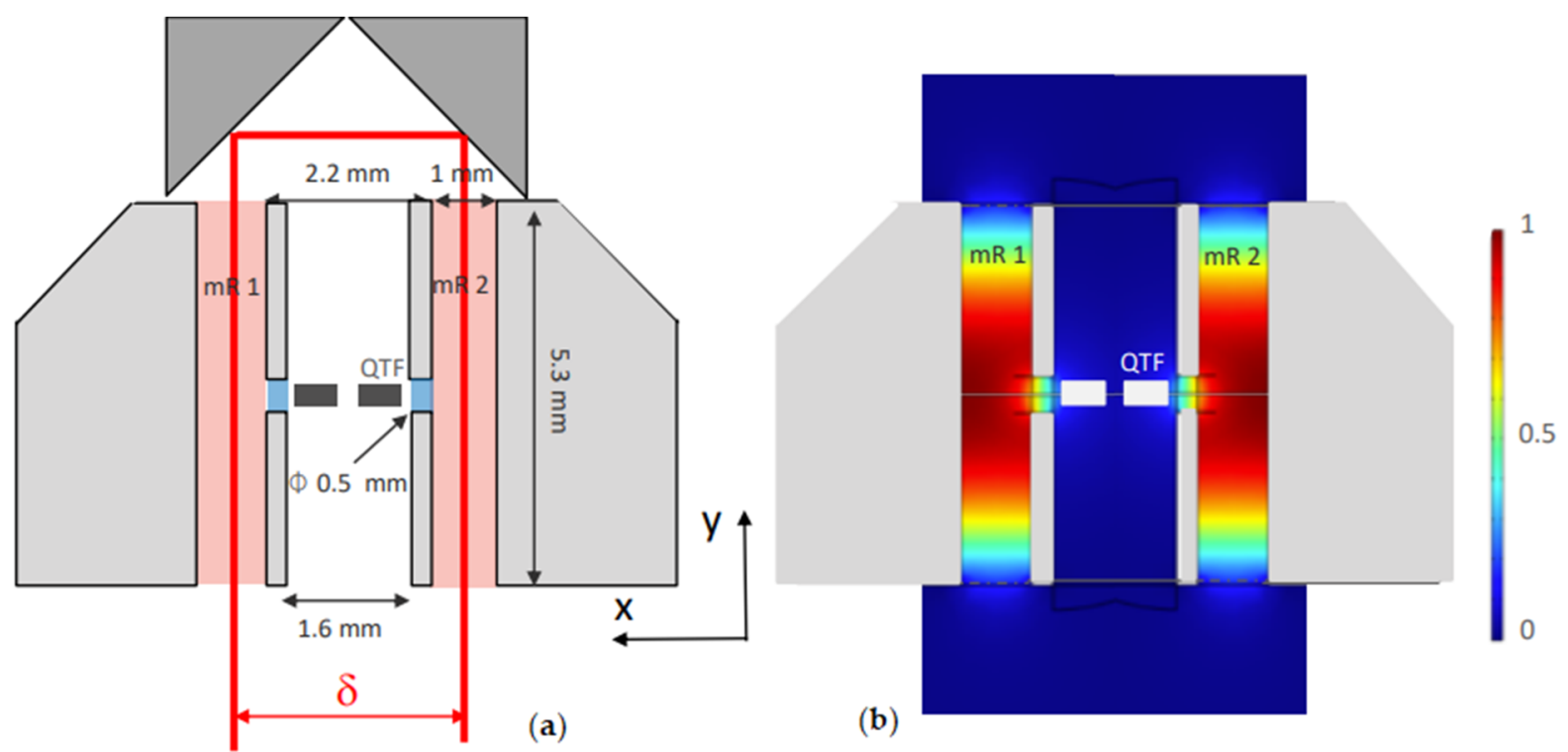

Figure 4. (a) Detailed dimensions of the monolithic double $\mathrm{mR}$. The laser beam is represented in red. It is reflected by the right angle mirrors. (b) Normalized acoustic pressure generated into the double $\mathrm{mR}$ for $\mathrm{f}=32,815 \mathrm{~Hz}$, corresponding to the QTF resonant frequency.

The setup was pre-aligned using a red laser. The visible laser was positioned instead of the IR laser and sent to $\mathrm{mR} 1$. The mirrors were translated until the exiting beam consistently crossed $\mathrm{mR} 2$. Next, the red laser was replaced by the IR laser and a focusing lens ( $f=4 \mathrm{~mm}$, Thorlabs C036TME-D). A power meter was used to monitor the output power. The exiting beam was sent to the power meter by using a tiny flat mirror, without cutting the incoming beam. The beam had to cross the $2 \mathrm{mRs}$ without impinging their wall, meaning the beam size width had to be smaller than the mR radius over a distance $\Delta L$ equal to $2.2 \mathrm{~cm}$. The laser was positioned about $20 \mathrm{~cm}$ far from the spectrophone to obtain a slowly converging beam. Using Gaussian optics and considering a beam waist of $1 \mu \mathrm{m}$ at the laser output and the given lens, it could be shown that the laser beam could cross the double $\mathrm{mR}$ without any reflection. Approximately half of the initial optical power was measured at the output. This value could be mainly explained by the contribution of direct absorption by ambient water vapor (for a path length of $25 \mathrm{~cm}$, and $50 \% \mathrm{RH}$, the power loss reached $36 \%$ ). The remaining losses could come from reflections on the aluminum mirrors. An xyz translation stage was used to accurately position the QTF in between the mRs. The QTF resonance was measured by means of electronic excitation and used as a control signal during the descent inside the monolithic double $\mathrm{mR}$ setup. This continuous control technique was necessary to prevent the QTF collision and breakage. The frequency responses of the QTF, free and inside the $\mathrm{mR}$, were compared, showing a $\mathrm{Q}$ factor of 14,140 and 9300, respectively (Figure 5). The $Q$ value decrease (of about 30\%) in this configuration was similar to the off-beam one. It was mainly due to the acoustic coupling between the QTF and $\mathrm{mR}$ [11]. The large signal offset observed in the characterization was due to the parallel capacitance of the coaxial cable connecting the transimpedance amplifier and could be easily suppressed. The parasitic capacitance could be suppressed by placing the amplifier closer to the QTF. 


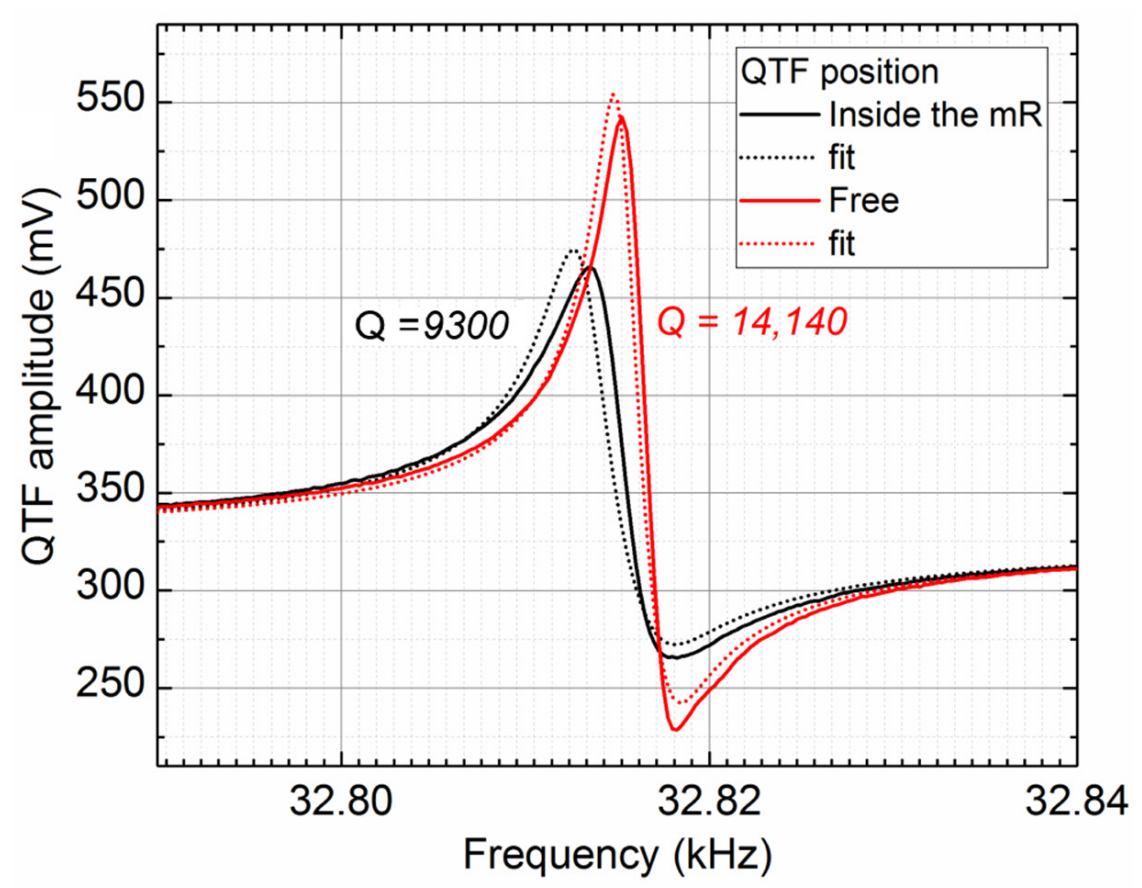

Figure 5. Frequency response of a QTF in free space (red) and positioned inside the double $\mathrm{mR}$ (black).

The $\mathrm{Q}$ factor was determined through the curve fitting with the Butterworth Von Dyke model.

\section{Experimental Evaluation}

The QTF was inserted in between the $\mathrm{mRs}$, and the laser beam crossed the two mRs. The QEPAS signal was monitored as a function of the QTF position, in the $y$ and $\mathrm{z}$ direction (Figure 6). The signal amplitude directly indicated the double mR-QTF coupling efficiency. In the $\mathrm{z}$ direction, the response grew significantly when the top of the prongs arrived in front of the slit and then decreased slowly as the lever became small. The response versus the $y$ direction exhibited a mirror symmetry with the ordinate axis, and a maximum when $\mathrm{y}=0$, when the prong and the $\mathrm{mR}$ slit best overlapped.

After the optimization of the QTF position, we studied the effect of each individual $\mathrm{mR}$ and compared it to the single off-beam configuration (with a single $\mathrm{mR}$ ). To this end, we first measured the QEPAS signal as a function of the mirrors position. The mirrors were translated in the perpendicular direction with respect to the incoming beam, using a micrometric translation stage, thus changing the value of $\delta$ (Figure 1a). At first, the laser beam only crossed the $\mathrm{mR} 1$, giving a QEPAS signal maximum of about $20 \mathrm{mV}$ (Figure 7). When the mirror was translated, the beam crossed the $\mathrm{mR} 2$ and the signal rose to $40 \mathrm{mV}$, doubling the initial value of the signal. The signal presented a plateau over $200 \mu \mathrm{m}$. The results could also be presented in terms of the QEPAS signal versus the laser current, with the typical $1 f$ signal shape (Figure $7 \mathrm{~b}$ ). We added the signal obtained with the conventional off-beam, whose amplitude was about $30 \mathrm{mV}$. The amplitude difference between the off-beam and the misaligned monolithic double $\mathrm{mR}$ setup was associated with the $\mathrm{mR}$ resonance. In the off-beam configuration, the slit was considered almost open while in the double $\mathrm{mR}$ configuration, the slit should be considered as almost closed, thus changing the acoustic resonant frequency. 

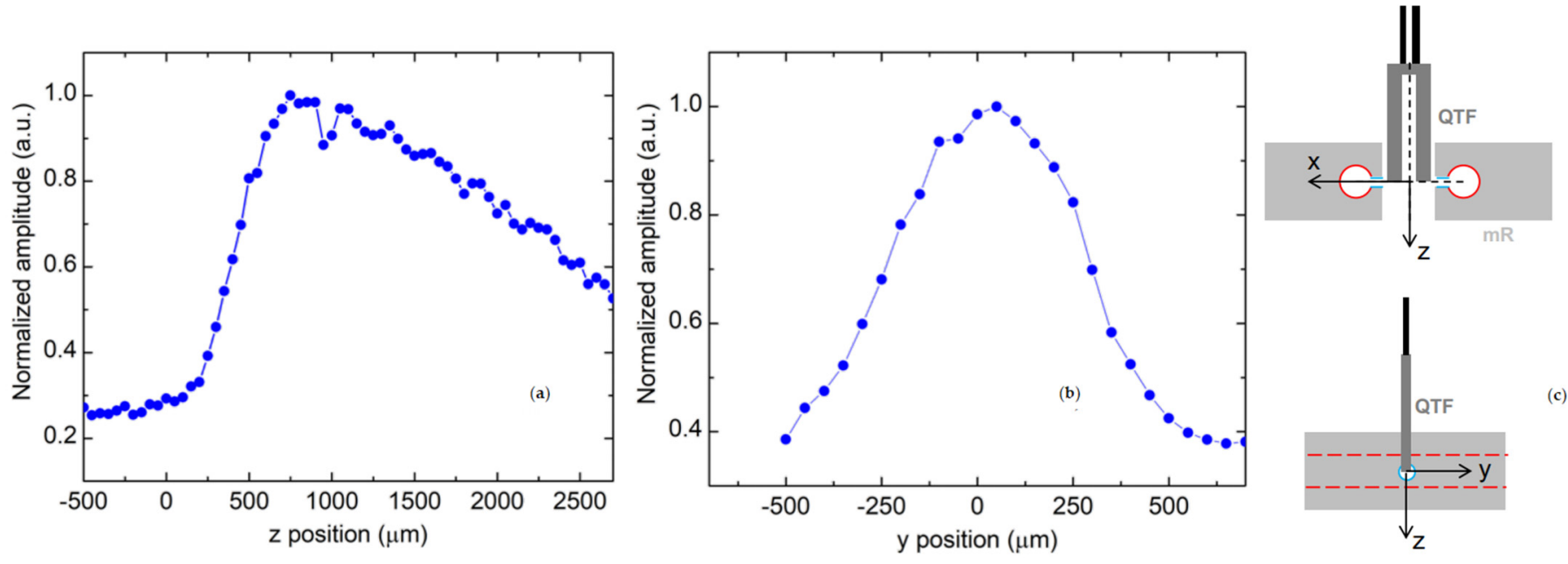

Figure 6. Evolution of the QEPAS signal when moving the QTF in the $\mathrm{z}(\mathbf{a})$ and $\mathrm{y}(\mathbf{b})$ direction. (c) Schematic of the $\mathrm{mR}$ and the QTF in the defined referential. The QTF is represented in the $(0,0,0)$ position.

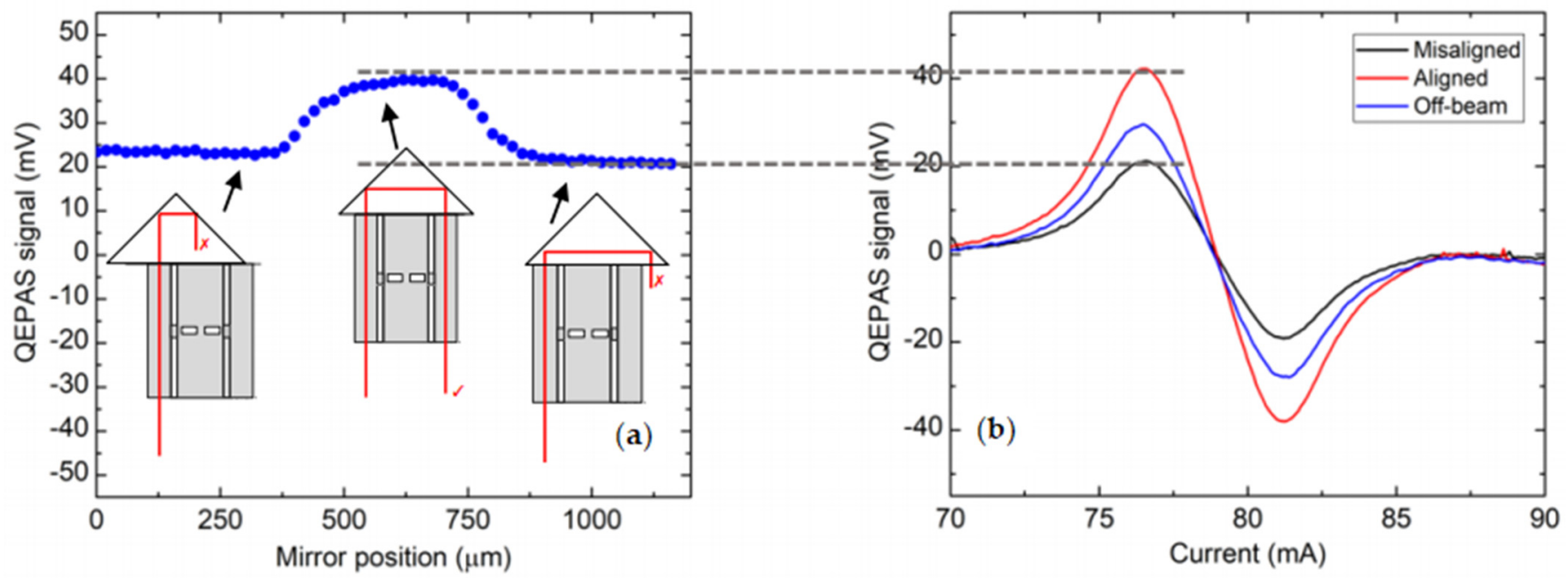

Figure 7. (a) Effect of the mirror position on the QEPAS signal maximum. (b) QEPAS signal versus laser current when the beam is misaligned (black) or aligned (red) with the $\mathrm{mR} \mathrm{2,} \mathrm{and} \mathrm{comparing} \mathrm{it} \mathrm{with} \mathrm{the} \mathrm{results} \mathrm{from} \mathrm{conventional}$ off-beam (blue).

$\mathrm{Hu}[16,17]$ made a thorough study on the effect of the length and the radius of the $\mathrm{mR}$. The optimized spectrophone showed a Signal to Noise Ratio (SNR) gain of $\approx 20$ and $\approx 40$, without and with the right-angle mirror, respectively, which corresponded to a two-factor increase, in good agreement with the results obtained in this paper.

We eventually estimated the normalized noise equivalent absorption (NNEA) on a 1f QEPAS signal in the double $\mathrm{mR}$ configuration (Figure 8a). We calculated the AllanWerle deviation (Figure $8 b$ ). For the given relative humidity and ambient temperature, the absolute water vapor concentration in ambient air was $9900 \mathrm{ppmv}\left(40 \% \mathrm{RH}, 20^{\circ} \mathrm{C}\right)$. The long-term drift appeared after a few seconds but the signal was really clean. The limit of detection was $2 \mathrm{ppmv}$ in $1 \mathrm{~s}$, giving an NNEA of $1.2 \times 10^{-8} \mathrm{~W} \cdot \mathrm{cm}^{-1} \cdot \mathrm{Hz}^{-0.5}$. 

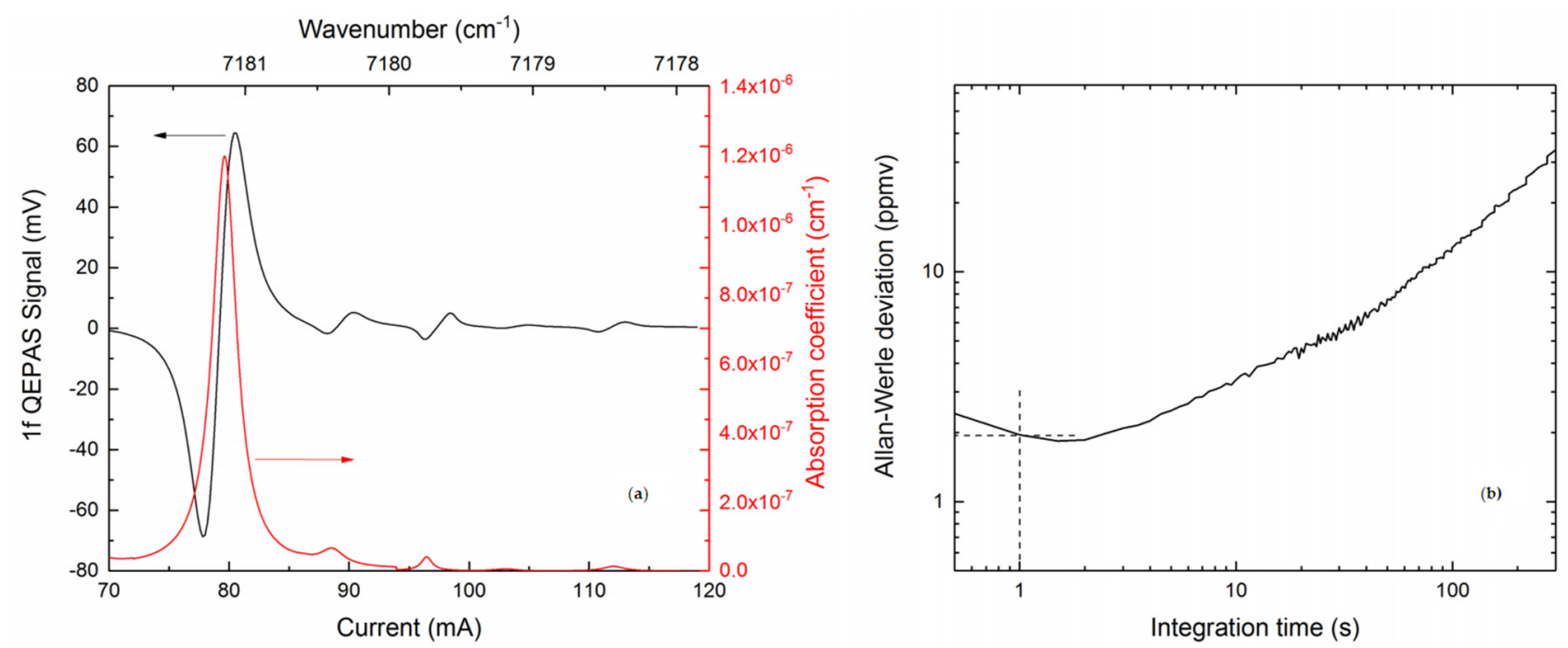

Figure 8. (a) 1f optimized double mR QEPAS signal (black). In red, the HITRAN database [18] absorption coefficient of the gas line (1ppmv) is reported. (b) Allan-Werle deviation calculated from a $15 \mathrm{~nm}$ acquisition of the 1f QEPAS signal.

The NNEA in this absorption range was comparable to a previously published paper [12] on water sensing, with a T-shape off-beam configuration. Even if the double $\mathrm{mR}$ if signal was clean, the long-term drift appeared sooner than in the QEPAS systems using closed cells. This value might be improved using a more stable configuration, with an enclosed gas cell.

\section{Conclusions}

A novel design was proposed, using $2 \mathrm{mRs}$ in a monolithic setup, to increase the acoustic pressure actuating on the two external sides of QTF prongs. The double $\mathrm{mR}$ was $3 \mathrm{D}$ printed, showing the ability and flexibility of the stereolithography method in printing small and complex parts.

While off-beam configuration might be less sensitive than on-beam, its advantages on the laser beam alignment and physics phenomena decoupling, offers many interests. The double $\mathrm{mR}$ spectrophone combine these advantages with a better acoustic excitation, thus, there is an increased value of the QEPAS signal. The strength of the double $\mathrm{mR}$ method relies on the large enhancement of the $\mathrm{mR}$ and QTF coupling. The surface of the prong was normal to the wave propagation direction, thus the acoustic pressure efficiently actuated the QTF. The weakness of the technique was the sensitivity to the QTF positioning, compared to the conventional off-beam. However, the required spatial accuracy $(10-30 \mu \mathrm{m})$ was definitely reachable, using micropositioners.

The gap between the $2 \mathrm{mRs}$ was adjusted at the printing level, simplifying the process of the QTF positioning. An enhancement could be obtained by adjusting the mRs dimensions to better fit the QTF frequency. It would be interesting to evaluate the use of custom QTF $[9,19]$ specifically developed to enhance coupling due to an external prong excitation. Finally, the high-resolution 3D-printing should be considered in the design of acoustic resonant cells, such as Helmholtz configurations coupled with mechanical resonators.

Author Contributions: R.R. was in charge of the methodology, the software, and the investigation, under the supervision of A.V. He was assisted by D.A., N.M. and W.T. for data curation. The article was written by R.R. and A.V., reviewed and edited by M.B. and A.V. All authors have read and agreed to the published version of the manuscript.

Funding: This research was funded by ANR MULTIPAS project (ANR-16-CE04-0012). It was partially supported by the French "Investment for the Future" program: EquipEx EXTRA, (ANR-11-EQPX0016) and Montpellier University MUSE SENSIR project (ANR-16-IDEX-0006).

Institutional Review Board Statement: Not applicable. 
Informed Consent Statement: Not applicable.

Data Availability Statement: Not applicable.

Conflicts of Interest: The authors declare no conflict of interest.

\section{References}

1. Kosterev, A.A.; Bakhirkin, Y.A.; Curl, R.F.; Tittel, F.K. Quartz-enhanced photoacoustic spectroscopy. Opt. Lett. 2002, $27,1902-1904$. [CrossRef] [PubMed]

2. Köhring, M.; Böttger, S.; Willer, U.; Schade, W. LED-absorption-QEPAS sensor for biogas plants. Sensors 2015, 15, 12092-12102. [CrossRef] [PubMed]

3. Palzer, S. Photoacoustic-based gas sensing: A review. Sensors 2020, 20, 2745. [CrossRef] [PubMed]

4. Weidmann, D.; Kosterev, A.A.; Tittel, F.K.; Ryan, N.; McDonald, D. Application of a widely electrically tunable diode laserto chemical gas sensing with quartz-enhanced photoacoustic spectroscopy. Opt. Lett. 2004, 29, 1837-1839. [CrossRef] [PubMed]

5. Liu, K.; Guo, X.; Yi, H.; Chen, W.; Zhang, W.; Gao, X. Off-beam quartz-enhanced photoacoustic spectroscopy. Opt. Lett. 2009, 34, 1594-1596. [CrossRef] [PubMed]

6. Dong, L.; Kosterev, A.A.; Thomazy, D.; Tittel, F.K. QEPAS spectrophones: Design, optimization, and performance. Appl. Phys. B 2010, 100, 627-635. [CrossRef]

7. Yi, H.; Chen, W.; Guo, X.; Sun, S.; Liu, K.; Tan, T.; Zhang, W.; Gao, X. An acoustic model for microresonator in on-beam quartz-enhanced photoacoustic spectroscopy. Appl. Phys. B 2012, 108, 361-367. [CrossRef]

8. Borri, S.; Patimisco, P.; Sampaolo, A.; Beere, H.E.; Ritchie, D.A.; Vitiello, M.S.; Scamarcio, G.; Spagnolo, V. Terahertz quartz enhanced photo-acoustic sensor. Appl. Phys. Lett. 2013, 103, 021105. [CrossRef]

9. Patimisco, P.; Sampaolo, A.; Zheng, H.; Dong, L.; Tittel, F.K.; Spagnolo, V. Quartz-enhanced photoacoustic spectrophones exploiting custom tuning forks: A review. Adv. Phys. X 2017, 2, 169-187. [CrossRef]

10. Yi, H.; Liu, K.; Sun, S.; Zhang, W.; Gao, X. Theoretical analysis of off beam quartz-enhanced photoacoustic spectroscopy sensor. Opt. Commun. 2012, 285, 5306-5312. [CrossRef]

11. Liu, K.; Yi, H.; Kosterev, A.A.; Chen, W.; Dong, L.; Wang, L.; Tan, T.; Zhang, W.; Tittel, F.K.; Gao, X. Trace gas detection based on off-beam quartz enhanced photoacoustic spectroscopy: Optimization and performance evaluation. Rev. Sci. Instrum. 2010, 81, 103103. [CrossRef] [PubMed]

12. Yi, H.; Chen, W.; Sun, S.; Liu, K.; Tan, T.; Gao, X. T-shape microresonator-based high sensitivity quartz-enhanced photoacoustic spectroscopy sensor. Opt. Express 2012, 20, 9187-9196. [CrossRef] [PubMed]

13. Rück, T.; Bierl, R.; Matysik, F. $\mathrm{NO}_{2}$ trace gas monitoring in air using off-beam quartz enhanced photoacoustic spectroscopy (QEPAS ) and interference studies towards $\mathrm{CO}_{2}, \mathrm{H}_{2} \mathrm{O}$ and acoustic noise. Sens. Actuators B Chem. 2018, 255, 2462-2471. [CrossRef]

14. Maurin, N.; Rousseau, R.; Trzpil, W.; Aoust, G.; Hayot, M.; Mercier, J.; Bahriz, M.; Gouzi, F.; Vicet, A. First clinical evalua-tion of a quartz enhanced photo-acoustic CO sensor for human breath analysis. Sens. Actuators B Chem. 2020, 128247. [CrossRef]

15. Rousseau, R.; Loghmari, Z.; Bahriz, M.; Chamassi, K.; Teissier, R.; Baranov, A.N.; Vicet, A. Off-beam QEPAS sensor using an 11- $\mu \mathrm{m}$ DFB-QCL with an optimized acoustic resonator. Opt. Express 2019, 27, 7435-7446. [CrossRef] [PubMed]

16. Hu, L.; Zheng, C.; Zheng, J.; Wang, Y.; Tittel, F.K. Quartz tuning fork embedded off-beam quartz-enhanced photoacoustic spectroscopy. Opt. Lett. 2019, 44, 2562. [CrossRef] [PubMed]

17. Hu, L.; Zheng, C.; Zhang, M.; Yao, D.; Zheng, J.; Zhang, Y.; Wang, Y.; Tittel, F.K. Quartz-enhanced photoacoustic spectroscopic methane sensor system using a quartz tuning fork-embedded, double-pass and off-beam configuration. Photoacoustics 2020, 100174. [CrossRef] [PubMed]

18. Gordon, I.E.; Rothman, L.S.; Hill, C.; Kochanov, R.V.; Tan, Y.; Bernath, P.F.; Zak, E.J. The HITRAN2016 molecular spectroscopic database. J. Quant. Spectrosc. Radiat. Transf. 2017, 203, 3-69. [CrossRef]

19. Duquesnoy, M.; Aoust, G.; Melkonian, J.M.; Lévy, R.; Raybaut, M.; Godard, A. Quartz enhanced photoacoustic spectroscopy based on a custom quartz tuning fork. Sensors 2019, 19, 1362. [CrossRef] [PubMed] 